\title{
The Self-Directed Learning Ability of Junior Nurses in the Emergency Department: An Up-to-date Survey
}

\begin{abstract}
Nie Fali ${ }^{1, \#}$ Han Xiaoling ${ }^{2, \# ~} \quad$ Sang Ziyang ${ }^{3} \quad$ Ye Xiaomei $^{4} \quad$ Zhong Yanhui $^{5} \quad$ Huang Xuanjie $^{6, *}$
${ }^{1}$ Emergency Department, Shenzhen Hospital of Southern Medical University, Shenzhen, Guangdong, China, 518110;

${ }^{2}$ Department of Nursing, Zhuhai People's Hospital (Zhuhai hospital affiliated with Jinan University), Zhuhai, Guangdong, China, 519000; ${ }^{3}$ Emergency Department, The Seventh Affiliated Hospital of Sun Yat-sen University, Shenzhen, Guangdong, China, 518107; ${ }^{4}$ Department of Intensive Care Unit, The Second Affiliated Hospital of Guangzhou Medical University. Guangzhou, Guangdong, China, 510220; ${ }^{5}$ Emergency Department, Shenzhen Hospital of Southern Medical University, Shen Zhen, Guangdong, China, 518110; ${ }^{6}$ Emergency Department, the Seventh Affiliated Hospital, Sun Yat-sen University, Shen Zhen, 518107; Xuanjie Huang, E-mail: huangxj67@mail.sysu.edu.cn, https://orcid.org/0000-0002-8811-7937; " Nie Fali and Han Xiaoling contributed to this study equally.
\end{abstract}

*Xuanjie Huang is the corresponding author.

Article History Received 25 January 2021 Accepted 25 January 2021 Published 31 March 2021

Cite this Article Nie Fali, Han Xiaoling, Sang Ziyang, Ye Xiaomei, Zhong Yanhui, Huang Xuanjie. Clinical The Self-Directed Learning Ability of Junior Nurses in the Emergency Department: An Up-to-date Survey [J].Medical Research, 2021.3(1):12-19, http://dx.doi.org/10.6913/MRHK.202103_3(1).0003

Copyright @ 2021 Creative Publishing Co., Limited. All rights reserved. Email:mrhk26640333@ gmail.com.

\section{ABSTRACT}

Objective This study aimed to evaluate the self-directed learning ability of junior emergency department (ED) nurses and provide the rationale for whether it is necessary to strengthen the training of junior ED nurses.

Methods Using the convenience sampling method, a self-directed learning ability questionnaire survey was conducted among 358 junior ED nurses in several tertiary hospitals in Guangdong Province.

Results The total score of self-learning ability of junior ED nurses was 119.85 \pm 16.82 (scores >135, 85-135, and $<85$ indicate excellent, intermediate, and poor self-learning ability, respectively). The retrospective analysis showed that gender, whether having participated in academic education, and the degree of motivation for promotion were major factors that affected the self-learning ability of junior ED nurses $(\mathrm{P}<0.05)$.

Conclusions The self-learning ability of junior ED nurses in Guangdong is at an intermediate level. Nursing managers should pay more attention to the junior ED nurses with poor self-learning ability. In particular, adopting various training methods and establishing appropriate promotion mechanisms and economic incentive measures may help improve their self-learning ability and enable them to meet job requirements.

Keywords Emergency department; Junior nurses; Self-directed learning; Influencing factors

\section{INTRODUCTION}

The emergency department (ED) is the first line of defense for the hospital to treat critically ill patients, and ED nurses play an important role in the rescues. However, in the emergency department of most 12

Copyright @ 2021 Creative Publishing Co., Limited. All rights reserved.mrhk26640333@gmail.com 
domestic hospitals, junior nurses with little experience account for a large proportion of ED nurses ${ }^{[1]}$. It has been reported that ED nurses at junior levels may have insufficient operational, critical thinking, proactive problem-solving skills that are required for critically ill patient care, which affects the overall core competence of ED nurses and the success rate of patient rescue ${ }^{[2]}$. Therefore, it is necessary to train the nurses at junior levels in the emergency department. However, the traditional training model for the junior ED nurses is mostly passive, which makes it difficult to achieve good training results ${ }^{[3]}$. The self-directed learning ability of nurses refers to the ability of nurses to take advantage of their subjective initiative to have a strong desire to learn knowledge actively, and particularly, to master the knowledge and skills necessary for nursing work with high quality ${ }^{[4]}$. The study aimed to evaluate the self-directed learning ability of junior ED nurses and provide a basis for guiding nursing managers to develop training plans suitable for junior ED nurses.

\section{MATERIALS AND METHODS}

\section{Research participants}

In March 2021, using a convenience sampling method, 358 junior ED nurses from a number of tertiary hospitals in Guangdong Province were enrolled in this study. The inclusion criteria were 1) having a nurse practitioner qualification certificate from the People's Republic of China, 2) working in the ED for over 6 months, and 3) the respondents agree to participate in this survey. The exclusion criteria included 1) those who could not complete the questionnaire in time due to study abroad, maternity leave, personal leave, etc., 2) those who did not actively cooperate and did not answer the questionnaire carefully, and 3) those who had been on the job for less than 6 months.

\section{General information collection form}

This form was a self-designed general information survey form for nurses. The collected information included gender, working years, education, job title, whether they are staff members, whether they have participated in academic education, monthly income, whether they are specialized nurses, their desire for promotion, and their marital status.

\section{Assessment tool for self-directed learning ability of junior ED nurses}

The self-directed learning ability evaluation scale developed by Shuqin Xiao from China Medical University (CMU) in 2008 was used for the evaluation ${ }^{[4]}$. This scale included four dimensions, namely self-motivation beliefs, task analysis, self-monitoring and adjustment, and self-regulation, with a total of 34 items. Using the Likert 5-level scoring system, "Strongly disagree", "Disagree", "Neither agree nor disagree", "Agree", "Strongly agree" are scored 1-5 points respectively; negative items are scored in reverse. The higher the score, the stronger the self-learning ability. A score $\geq$ of 136 indicated excellent self-directed learning ability, a score between 85-135 is indicated moderate self-directed learning ability, and a score $\leq$ of 84 indicated poor self-learning ability. The Cronbach's Alpha Reliability Coefficient of this scale is 0.944 .

\section{Data collection method}


Before starting this investigation, the consent and cooperation of the nursing staff in the ED of each hospital were obtained. The number of questionnaires to be issued and the survey time were determined according to the specific conditions of the ED of different hospitals. The researcher stated the purpose of the survey before the questionnaire started. After obtaining the informed consent of the research subjects, the respondents began to fill out the questionnaire (through an online platform). A total of 358 questionnaires were returned, all of which were valid questionnaires, with an effective rate of $100 \%$.

\section{Statistical analysis}

The statistical analysis was carried out using SPSS 24.0 software. The data of general information of junior ED nurses were described by frequency and percentage. Measurement data were expressed as mean and standard deviation (mean $\pm \mathrm{SD}$ ). Logistic regression analysis was used to analyze related factors. A p-value of less than 0.05 indicated that the difference was statistically significant.

\section{RESULT}

\section{The general information of the junior ED nurses}

As shown in Table 1, among the junior ED nurses who participated in the questionnaire ( $n=358), 184$ (51.4\%) had a junior college degree. Most junior ED nurses (60.3\%) had job titles as nurses. There were 178 people (49.7\%) with 3-5 years of work experience, and 180 people $(51.3 \%)$ with less than 3 years of work experience. Most of them were contractors with strong motivation for promotion.

Table 1. The general information of the junior ED nurses

\begin{tabular}{ccc}
\hline & Items & N (\%) \\
\hline \multirow{2}{*}{ Gender } & Male & $68(19.0)$ \\
& Female & $290(81.0)$ \\
\hline \multirow{2}{*}{ Working experience } & $<1$ year & $44(12.3)$ \\
& $1-2$ years & $76(21.2)$ \\
& $2-3$ years & $60(16.8)$ \\
& 3-5 years & $178(49.7)$ \\
\hline \multirow{2}{*}{ Job title } & Assistant nurse & $24(6.7)$ \\
& Nurse & $216(60.3)$ \\
& Senior nurse & $100(27.9)$ \\
& Supervisor nurse & $18(5.1)$ \\
\hline \multirow{2}{*}{ Degree } & Technical secondary school & $27(7.5)$ \\
& Junior College & $184(51.4)$ \\
& Bachelor & $146(40.8)$ \\
Motivation for promotion & Master or higher & $1(0.28)$ \\
& Very strong & $120(33.5)$ \\
& Strong & $130(36.3)$
\end{tabular}




\begin{tabular}{ccc} 
& Not very strong & $104(29.1)$ \\
& None & $4(1.1)$ \\
\hline \multirow{2}{*}{ Full-time employee } & Yes & $24(6.7)$ \\
& No & $334(93.3)$ \\
\hline \multirow{2}{*}{ Participated in academic education } & Yes & $228(63.7)$ \\
& Never & $64(17.9)$ \\
& No, but plan to & $55(15.4)$ \\
& No, and not plan to & $11(3.1)$ \\
\hline \multirow{2}{*}{ Marital status } & Married & $100(27.9)$ \\
& Unmarried & $256(71.5)$ \\
\hline
\end{tabular}

The score of self-directed learning ability evaluation scale

The average score of the self-directed learning ability evaluation scale for 358 junior ED nurses was $118.99 \pm 19.55$. The scores of each dimension were shown in Table 2 .

Table 2. The self-directed learning ability evaluation scale score of junior ED nurses

\begin{tabular}{ccc}
\hline Item & Item numbers & Score (Mean \pm SD) \\
\hline Self-motivation beliefs & 14 & $50.3 \pm 8.39$ \\
Task analysis & 6 & $20.08 \pm 4.06$ \\
Self-monitoring and adjustment & 10 & $34.67 \pm 6.19$ \\
Self-regulation & 4 & $13.94 \pm 2.47$ \\
In total & 34 & $118.99 \pm 19.55$ \\
\hline
\end{tabular}

The self-directed learning ability among junior ED nurses with different characteristics

The comparison of self-directed learning ability among junior ED nurses with different characteristics is shown in Table 3 and the assignment of independent variables is detailed in Table 4.

Table 3. The comparison of self-directed learning ability among junior ED nurses with different characteristics

\begin{tabular}{ccccc}
\hline & Items & Scores & t/F value & P value \\
\hline \multirow{2}{*}{ Gender } & Male & $123.24 \pm 19.17$ & 4.122 & 0.042 \\
& Female & $117.99 \pm 19.54$ & & \\
\hline \multirow{2}{*}{ Working experience } & <1 year & $122.89 \pm 18.33$ & 2.564 & 0.464 \\
& 1-2 years & $119.34 \pm 21.2$ & & \\
& 2-3 years & $119.78 \pm 20.35$ & & \\
& 3-5 years & $117.6 \pm 18.84$ & & \\
& Technical secondary school & $116.41 \pm 18.2$ & 3.715 & 0.294 \\
Degree & Junior College & $118.51 \pm 17.87$ & & \\
& Bachelor & $120.23 \pm 21.71$ & &
\end{tabular}


Nie Fali,et.,al. Clinical The Self-Directed Learning Ability of Junior Nurses in the Emergency Department: An Up-to-date Survey Medical Research ISSN 2664-0333 eISSN 2664-0341 Volume 3 Issue 1 http://dx.doi.org/10.6913/MRHK.202103_3(1).0003

\begin{tabular}{|c|c|c|c|c|}
\hline & Master or higher & $94 \pm \mathrm{NA}^{*}$ & & \\
\hline \multirow{4}{*}{ Job title } & Assistant nurse & $123.75 \pm 15.21$ & 2.865 & 0.413 \\
\hline & Nurse & $118.95 \pm 19.42$ & & \\
\hline & Senior nurse & $118.47 \pm 21.52$ & & \\
\hline & Supervisor nurse & $115.89 \pm 14.26$ & & \\
\hline \multirow{2}{*}{ Full-time employee } & Yes & $116.38 \pm 17.56$ & 0.683 & 0.409 \\
\hline & No & $119.17 \pm 19.7$ & & \\
\hline \multirow{4}{*}{ Participated in academic education } & Yes & $120.52 \pm 18.81$ & 15.266 & 0.002 \\
\hline & Never & $114.7 \pm 18.46$ & & \\
\hline & No, but Plan to & $120.67 \pm 21.49$ & & \\
\hline & No, and not plan to & $103.73 \pm 23.05$ & & \\
\hline \multirow{4}{*}{ Income (¥) } & $<5000$ & $118.98 \pm 18.84$ & 3.126 & 0.373 \\
\hline & $5000-10000$ & $119.96 \pm 20.12$ & & \\
\hline & $10000-15000$ & $116.07 \pm 18.59$ & & \\
\hline & $15000-20000$ & $110.6 \pm 16.83$ & & \\
\hline \multirow{2}{*}{ Specialist nurse } & Yes & $117.35 \pm 22.22$ & 0.12 & 0.729 \\
\hline & No & $119.14 \pm 19.31$ & & \\
\hline \multirow{4}{*}{ Motivation for promotion } & Very strong & $124.47 \pm 21.59$ & 35.741 & $<0.001$ \\
\hline & Strong & $120.89 \pm 17.07$ & & \\
\hline & Not very strong & $110.39 \pm 17.24$ & & \\
\hline & None & $115.75 \pm 18.21$ & & \\
\hline \multirow{3}{*}{ Marital status } & Married & $118.96 \pm 19.56$ & 1.848 & 0.397 \\
\hline & Unmarried & $119.35 \pm 19.67$ & & \\
\hline & Others & $103.5 \pm 9.19$ & & \\
\hline
\end{tabular}

*Note: Only one person was in this category, so the SD value was not available.

Table 4. Assignment of independent variables

\begin{tabular}{cc}
\hline Variables & Assignment method \\
\hline Gender & Male=1; Female=2 \\
$\begin{array}{cc}\text { Participated in academic education } \\
\text { Motivation for promotion }\end{array}$ & Yes=1; No=2; No but plan to=3; No and not plan to=4 \\
\hline
\end{tabular}

\section{DISCUSSION}

Junior ED nurses need to improve their self-directed learning ability

In this study, the self-directed learning ability score of junior ED nurses is $118.99 \pm 19.55$, indicating that self-directed learning ability of junior ED nurses in Guangdong Province is at an intermediate level. This score is lower than the scores in the research reported by Pingping Zhuang et al. ${ }^{[5]}$, Xiaolan Diao et al. ${ }^{[6]}$, and Chen Qian et al. ${ }^{[7]}$ suggested that self-directed learning ability of junior ED nurses needs to be improved. 16 
Nie Fali,et.,al. Clinical The Self-Directed Learning Ability of Junior Nurses in the Emergency Department: An Up-to-date Survey Medical Research ISSN 2664-0333 eISSN 2664-0341 Volume 3 Issue $1 \quad$ http://dx.doi.org/10.6913/MRHK.202103_3(1).0003

Regarding the scores of four dimensions, the score of self-motivation belief was the highest, while the score of self-evaluation was the lowest; these results were consistent with the research results by Jiannv Wang et al ${ }^{[8]}$. Junior ED nurses may relate the reason for this score to the lack of monitoring of learning. Although they have a strong belief in learning, they lack the relevant experience in effectively learning professional knowledge since they have just entered this field. Similarly, the study by Jiannv Wang et al. has pointed out that junior nurses at the initial stage of career development are usually inexpensive and may often lack critical thinking skills to adjust learning strategies timely ${ }^{[8]}$. Therefore, emergency care managers may need to help improve their independent learning ability (for instance, setting learning goals) and supervise the learning efficiency of junior ED nurses.

Analysis of the influencing factors of the self-learning ability of the junior nurses in the emergency department

As shown in Table 3, gender, whether they have participated in academic education, and the degree of willingness to promote professional titles are the major factors affecting the self-directed learning ability of junior ED nurses $(\mathrm{p}<0.05)$.

Male junior ED nurses seem to have stronger self-directed learning abilities in comparison to female junior ED nurses

As shown in Table 3, the self-directed learning ability of male junior ED nurses is higher than that of female junior ED nurses. The reasons for this discrepancy are as follows. First, emergency work is intense and fast-paced. Male nurses might be physically and psychologically more competent than female nurses in emergency department work. Also, many hospitals tend to hire male nurses in the emergency department when recruiting nurses. However, in the traditional Chinese viewpoint, the nursing profession is full of prejudice and discrimination against men ${ }^{[9]}$. To get rid of this kind of prejudice and discrimination, male nurses tend to have a strong enterprising spirit to be successful in their careers. This mindset may drive them to learn actively, have better independent learning ability, and have a relatively clear career development plan. On the other hand, nurses with a clear career plan tend to have a high self-directed learning ability since clear goals can guide them to keep learning ${ }^{[10]}$.

Junior ED nurses who have participated in academic education have a stronger self-learning ability than those who have not

This study also shows that junior ED nurses who have participated in academic education are more capable of autonomous learning, which is consistent with the results of Cunmei Tan et al [11]. Among the research participants, 211 people (58.9\%) had a college degree or a technical secondary school degree. As undergraduate education is becoming more and more popular today, undergraduate degree is the basic educational requirement for nurses' promotion and further training. Therefore, a large number of nurses promote themselves through continuing education. They have shown their ability to pass the exam successfully, and getting a diploma has been a learning goal for them. To reach the goal, they need to formulate a specific study plan, and the school's requirements for the curriculum have played a monitoring 
and regulating role. These learning experiences of junior ED nurses post-employment may improve their independent learning ability, making them superior to the junior ED nurses in terms of self-directed learning ability.

\section{Junior ED nurses with a strong desire for promotion have strong self-learning ability}

This study shows that junior ED nurses who have a strong desire for promotion have better learning ability. This is consistent with the previous results showing that nurses with good self-learning ability are more likely to pass the qualification examination and be promoted smoothly ${ }^{[7]}$. In most hospitals, the higher the professional title, the better the corresponding treatments, and the more opportunities for studying and training; these opportunities are the reasons why many people want to be promoted. On the other hand, the promotion of the professional title itself can be a goal. The promotion requires junior ED nurses to self-monitor and adjust and requires them to actively learn relevant knowledge and meet the promotion requirements in theory and scientific research. Therefore, to a certain extent, the desire for promotion helps improve their independent learning ability.

\section{Strategies to improve the self-directed learning ability of junior ED nurses}

The results of this study show that the self-directed learning ability of junior ED nurses is greatly affected by the three factors, including gender, whether they have participated in academic education, and the desire to be promoted. Therefore, starting from these three aspects and formulating relevant practical strategies are critical to improving their independent learning ability. First, nursing managers can work on the shift schedule of nurses, arranging the nurses with different genders, professional titles, and educational backgrounds in a better way that they can have positive influence on each other in their daily work. Secondly, different training programs can be introduced to junior ED nurses with different requirements and different levels. Particularly, the training programs, either through modeling rewards and incentive mechanisms ${ }^{[12]}$ or psychological interventions ${ }^{[13]}$ that can help them formulate clear career development plans and continuing education plans are beneficial. Third, setting small goals and appropriate financial rewards, as well as proper supervising, are critical; junior ED nurses could gradually develop the self-directed learning ability in the process of achieving their goals.

\section{CONCLUSION}

The self-learning ability of junior ED nurses in Guangdong Province is at an intermediate level. Nursing managers should pay more attention to the ED nurses with poor self-learning ability. In particular, adopting various training methods and establishing appropriate promotion mechanisms and economic incentive measures may help improve their self-learning ability and enable them to meet job requirements.

\section{ACKNOWLEDGMENT}

None.

\section{STATEMENT}

There is no conflict of interest in this article. 


\section{REFERENCES}

1 Yingli Hu; Jingya Huang; Lina Qiu; Yaran Ming. Grade-based nursing continuing education program for junior nurses in emergency department: evaluation of the effect [J]. JOURNAL OF NURSING SCIENCE, 2013, 28(21):4-6.

2 Shuxia Li. Cultivation and Practice of Critical Thinking Ability of Junior Nurses in Emergency Department [J]. Chinese Journal of Rural Medicine and Pharmacy, 2014, 21(03):75+77.

3 James MS, Clancy DDS. A comparison of student performance in a simulation clinic and a traditional laboratory environment:Three year results[J]. J Dent Edue, 2002, 66(12):1331-1337.

4 Shuqin Xiao, Xiaohan Li. Development of a Rating Scale of Self-directed Learning Competence for Nurses [D]. JOURNAL OF NURSING SCIENCE, 2008, 23(20):1-4.

5 Ping Zhuang, Mei-zhen Lin, Guang-qing Zhang, Jiu-hong You, Xiao-pei Zhang. The level and influencing factors of self-directed learning ability of specialized nurses in Traditional Chinese Medicine hospital [J]. Journal of Nursing Administration, 2015, 15(02):83-85.

6 Xiao-lan Diao, Li-xian Liu, Xia Ye. Analysis of the independent learning and influencing factors of nurses in standardized training [J]. Modern Preventive Medicine, 2012, 39(15):4060-4062.

7 Chen Qian, Aihong Wang, Qian Ma. Correlation study between self-directed learning ability and core competency of operating room nurses [D]. Chinese Journal of Modern Nursing, 2016, 22(8):1071-1075.

8 Jiannv Wang, Qiuping Zhang, Yafen Lian, Xiaoying Zhu. Status and influencing factors of autonomous learning ability of 904 nurses in Class III Grade A psychiatric hospitals in Zhejiang Province [J]. Chinese Journal of Modern Nursing, 2020, 26(29):4033-4037.

9 Cheng M L, Tseng Y H, Hodges E, et al. Lived experiences of novice male nurses in Taiwan [J]. Journal of Transcultural Nursing, 2018, 29(1): 46-53.

10 Yongxia Ju,Xiaomin Zhao. A Qualitative Study on the Obstacle Factors of Self-directed Learning Ability in Clinical Nurses [J]. Chinese and Foreign Medical Research, 2019, 17(4):170-172.

11 Cunmei Tan, Haiyan Qi, Julan Yang. Investigation of self-directed learning ability in nurses [J]. Chinese Journal of Nursing Education, 2013, 10(08):371-373.

12 Yuan Xu. Application of a new model incentive mechanism in improving nurses' autonomous learning ability [J]. Journal of Clinic Nursing's Practicality, 2020, 5(17):177-178.

13 Fang Zou, Li Xiong. Observation on the effect of psychological intervention on improving the self-learning ability of clinical nurses [J]. Journal of Shenyang Medical College, 2015, 17(03):144-145+154. 